\title{
AN ELEMENTARY THEORY OF HYPERFUNCTIONS AND MICROFUNCTIONS
}

\author{
HIKOSABURO KOMATSU \\ Department of Mathematics, Faculty of Science, University of Tokyo \\ Tokyo, 113 Japan
}

1. Sato's definition of hyperfunctions. The hyperfunctions are a class of generalized functions introduced by M. Sato [34], [35], [36] in 1958-60, only ten years later than Schwartz' distributions [40]. As we will see, hyperfunctions are natural and useful, but unfortunately they are not so commonly used as distributions. One reason seems to be that the mere definition of hyperfunctions needs a lot of preparations.

In the one-dimensional case his definition is elementary. Let $\Omega$ be an open set in $\mathbb{R}$. Then the space $\mathcal{B}(\Omega)$ of hyperfunctions on $\Omega$ is defined to be the quotient space

$$
\mathcal{B}(\Omega)=\mathcal{O}(V \backslash \Omega) / \mathcal{O}(V),
$$

where $V$ is an open set in $\mathbb{C}$ containing $\Omega$ as a closed set, and $\mathcal{O}(V \backslash \Omega)$ (resp. $\mathcal{O}(V)$ ) is the space of all holomorphic functions on $V \backslash \Omega$ (resp. $V$ ). The hyperfunction $f(x)$ represented by $F(z) \in \mathcal{O}(V \backslash \Omega)$ is written

$$
f(x)=F(x+i 0)-F(x-i 0)
$$

and has the intuitive meaning of the difference of the "boundary values" of $F(z)$ on $\Omega$ from above and below.

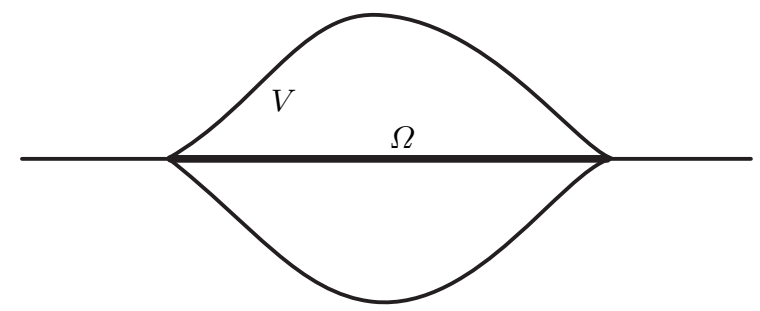

Fig. 1 
The main properties of hyperfunctions are the following:

(1) $\mathcal{B}(\Omega), \Omega \subset \mathbb{R}$, form a sheaf over $\mathbb{R}$.

Namely, for all pairs $\Omega_{1} \subset \Omega$ of open sets the restriction mappings $\varrho_{\Omega_{1}}^{\Omega}$ : $\mathcal{B}(\Omega) \rightarrow \mathcal{B}\left(\Omega_{1}\right)$ are defined, and for any open covering $\Omega=\bigcup \Omega_{\alpha}$ they satisfy the following conditions:

$$
\text { If } f \in \mathcal{B}(\Omega) \text { satisfies }\left.f\right|_{\Omega_{\alpha}}=0 \text { for all } \alpha \text {, then } f=0 \text {; }
$$

(S.2) If $f_{\alpha} \in \mathcal{B}\left(\Omega_{\alpha}\right)$ satisfy $\left.f_{\alpha}\right|_{\Omega_{\alpha} \cap \Omega_{\beta}}=\left.f_{\beta}\right|_{\Omega_{\alpha} \cap \Omega_{\beta}}$ for all $\Omega_{\alpha} \cap \Omega_{\beta} \neq \emptyset$, then there is an $f \in \mathcal{B}(\Omega)$ such that $f_{\alpha}=\left.f\right|_{\Omega_{\alpha}}$.

(2) The sheaf $\mathcal{B}$ of hyperfunctions is flabby.

That is, the restriction mappings $\varrho_{\Omega_{1}}^{\Omega}$ are always surjective.

By property (S.1) each $f \in \mathcal{B}(\Omega)$ has the maximal open subset of $\Omega$ on which it vanishes. Its complement is called the support of $f$ and is denoted by $\operatorname{supp} f$. If $F$ is a closed set in $\Omega$, we write

$$
\mathcal{B}_{F}(\Omega)=\{f \in \mathcal{B}(\Omega) ; \operatorname{supp} f \subset F\} .
$$

(3) If $K$ is a compact set in $\Omega$, then

$$
\mathcal{B}_{K}(\Omega)=\mathcal{A}(K)^{\prime},
$$

where the right hand side denotes the space of all continuous linear functionals on the locally convex space

$$
\mathcal{A}(K)=\underset{\overrightarrow{V \supset K}}{\lim } \mathcal{O}(V)
$$

of all germs of real-analytic functions defined on a neighborhood of $K$. The pairing is given by the integral

$$
\langle\varphi,[F]\rangle=-\oint_{\Gamma} \varphi(z) F(z) d z,
$$

where $\varphi \in \mathcal{A}(K), F \in \mathcal{O}(V \backslash K)$ and $\Gamma$ is a closed curve in the intersection of the domain of $\varphi$ and $V \backslash K$, which encircles $K$ once.

These three properties characterize the sheaf $\mathcal{B}$ of hyperfunctions. In the onedimensional case they are derived from the Köthe duality [26]

$$
\mathcal{A}(K)^{\prime} \cong \mathcal{O}(V \backslash K) / \mathcal{O}(V),
$$

and the Mittag-Leffler theorem

$$
H^{1}(V, \mathcal{O})=0 \quad \text { for any open set } V \text { in } \mathbb{C} .
$$

The hyperfunctions in the higher dimensional case have the same properties, and are characterized by them, too. It was not easy, however, to define the spaces $\mathcal{B}(\Omega), \Omega \subset \mathbb{R}^{n}$, of hyperfunctions having these properties. Sato had spent two years before he succeeded in giving a definition for the higher dimensional case. His definition is

$$
\mathcal{B}(\Omega)=H^{n}(V, V \backslash \Omega ; \mathcal{O}),
$$


where the right hand side is the $n$th relative cohomology group of the open pair $(V, V \backslash \Omega)$ with coefficients in the sheaf $\mathcal{O}$ of holomorphic functions, which he invented for this purpose. The same concept was independently introduced by Grothendieck [12] under the name of the local cohomology group with support in $\Omega$. In Grothendieck's notation it is written $H_{\Omega}^{n}(V, \mathcal{O})$.

In the earliest foundation of the theory of hyperfunctions, Martineau [30] and Harvey [13] (cf. Komatsu [22]) derived the three properties from the Martineau duality

$$
H_{K}^{p}(V, \mathcal{O})= \begin{cases}0, & p \neq n, \\ \mathcal{A}(K)^{\prime}, & p=n,\end{cases}
$$

for any compact set $K$ in $\mathbb{R}^{n}$ included in an open set $V$ in $\mathbb{C}^{n}$, the Malgrange theorem [28]

$$
H^{n}(V, \mathcal{O})=0 \quad \text { for any open set } V \text { in } \mathbb{C}^{n}
$$

and the Grauert theorem [10] saying that for any open set $\Omega$ in $\mathbb{R}^{n}$ its pseudoconvex open neighborhoods $V$ in $\mathbb{C}^{n}$ form a fundamental system of neighborhoods.

The disadvantage of this definition is that one has to take a one-year course of several complex variables and homological algebra before he understands the fundamental concepts.

2. Hyperfunctions as boundary values of harmonic functions. As we remarked, the hyperfunctions are natural and useful. One evidence is that they are closed under taking non-characteristic boundary values of solutions of linear partial differential equations. Namely, let

$$
P(x, \partial)=\sum_{|\alpha| \leq m} a_{\alpha}(x) \partial^{\alpha}
$$

be a partial differential operator with real-analytic coefficients $a_{\alpha}(x)$ on an open set $V$ in $\mathbb{R}^{n+1}$, and let $\Omega=V \cap \mathbb{R}^{n}$ be a non-characteristic hypersurface, or $a_{(0, \ldots, 0, m)}\left(x^{\prime}, 0\right) \neq 0$. Then we have

$$
H_{\Omega}^{1}\left(V, \mathcal{B}^{P}\right) \cong \mathcal{B}(\Omega)^{m}
$$

(Komatsu [21], Schapira [39] and Komatsu-Kawai [25]). If $P$ has constant coefficients, then

$$
H_{\Omega}^{1}\left(V, \mathcal{B}^{P}\right) \cong \mathcal{B}^{P}(V \backslash \Omega) / \mathcal{B}^{P}(V) .
$$

Here $\mathcal{B}^{P}$ denotes the sheaf of hyperfunction solutions $u$ of $P u=0$. The isomorphisms (2.2) and (2.3) mean that the right hand side of (2.3) is identified with the $m$-tuples of hyperfunctions $\left(\partial_{n}^{j} u\left(x^{\prime},+0\right)-\partial_{n}^{j} u\left(x^{\prime},-0\right)\right)_{0 \leq j<m}$ in $n$ variables. This is proved only by properties (1), (2) and (3) of hyperfunctions and by the Cauchy-Kowalevsky theorem.

If $P(\partial)$ is elliptic, then the hyperfunction solutions $\mathcal{B}^{P}$ are real-analytic and hence we may replace $\mathcal{B}^{P}$ in $(2.2)$ and $(2.3)$ by the sheaf $\mathcal{A}^{P}$ of real-analytic 
solutions. If $n=1$, the Cauchy-Riemann operator

$$
\bar{\partial}=\frac{1}{2}\left(\partial_{x}+i \partial_{y}\right)
$$

is an elliptic operator. In case $n>1$, there are no single elliptic operators of first order. The Laplacian $\Delta$ would be the simplest elliptic operator in that case.

We denote points in $\mathbb{R}^{n+1}$ by $v=(x, t), w=(y, s)$ with $x, y \in \mathbb{R}^{n}$ and $t, s \in \mathbb{R}$, and the sheaf of harmonic functions on $\mathbb{R}^{n+1}$ by $\mathcal{P}$. Thus we have for any open set $V$ in $\mathbb{R}^{n+1}$

$$
\mathcal{P}(V)=\left\{H(v) \in C^{2}(V) ; \Delta H=\left(\Delta_{x}+\partial_{t}^{2}\right) H=0\right\} .
$$

Let $\Omega=V \cap \mathbb{R}^{n}=\left\{x \in \mathbb{R}^{n} ;(x, 0) \in V\right\}$. Then with any $H(v) \in \mathcal{P}(V \backslash \Omega)$, two boundary values

$$
H(x,+0)-H(x,-0) \in \mathcal{B}(\Omega), \quad \partial_{t} H(x,+0)-\partial_{t} H(x,-0) \in \mathcal{B}(\Omega)
$$

are associated and $H(v)$ can be continued to a harmonic function on $V$ if and only if these boundary values vanish. Moreover, all pairs of hyperfunctions on $\Omega$ appear as boundary values.

If we take an open set $V$ symmetric in $t$, then every $H \in \mathcal{P}(V \backslash \Omega)$ is decomposed into the sum of the odd harmonic function

$$
H_{-}(x, t)=\frac{1}{2}(H(x, t)-H(x,-t))
$$

and the even one

$$
H_{+}(x, t)=\frac{1}{2}(H(x, t)+H(x,-t)) .
$$

Clearly the Neumann data (resp. the Dirichlet data) vanishes for $H_{-}$(resp. $H_{+}$). Therefore every $f(x) \in \mathcal{B}(\Omega)$ is represented as the Dirichlet boundary value of an odd harmonic function $H_{-}$. Moreover, the Dirichlet boundary value of $H_{-}$ vanishes if and only if it can be extended to an odd harmonic function on $V$. Thus we have the representation

$$
\mathcal{B}(\Omega)=\mathcal{P}_{-}(V \backslash \Omega) / \mathcal{P}_{-}(V),
$$

where $\mathcal{P}_{-}$denotes the space of odd harmonic functions.

If we admit properties (1), (2) and (3) of hyperfunctions, this is a theorem. Here we make this representation the definition of hyperfunctions and derive the properties (1), (2) and (3) as theorems.

For the sake of convenience we write the upper half of $V$ as $V$, the original $V$ as $\widetilde{V}$ and $\mathcal{P}_{-}(V)$ as $\mathcal{P}_{0}(\widetilde{V})$, and consider only the one-sided limit.

Definition 1. Let $\Omega$ be an open set in $\mathbb{R}^{n}$, and $V$ an open set in $\mathbb{R}_{+}^{n+1}=$ $\left\{(x, t) \in \mathbb{R}^{n+1} ; t>0\right\}$ such that $\widetilde{V}=V \cup \Omega \cup \bar{V}$ is open in $\mathbb{R}^{n+1}$, where $\bar{V}$ is the mirror image of $V$ :

$$
\bar{V}=\left\{(x, t) \in \mathbb{R}^{n+1} ;(x,-t) \in V\right\} .
$$

Then the space $\mathcal{B}(\Omega)$ of hyperfunctions on $\Omega$ is defined to be the quotient space

$$
\mathcal{B}(\Omega)=\mathcal{P}(V) / \mathcal{P}_{0}(\widetilde{V}) .
$$


If a hyperfunction $f(x) \in \mathcal{B}(\Omega)$ is represented by a harmonic function $H(x, t) \in$ $\mathcal{P}(V)$, we write

$$
f(x)=H(x,+0) .
$$

The basic theorems we need are the following two.

The Grothendieck DuALity theorem $([11],[29])$. Let $K$ be a compact set in an open set $V$ in $\mathbb{R}^{n+1}$. Then

$$
\mathcal{P}(K)^{\prime} \cong \mathcal{P}(V \backslash K) / \mathcal{P}(V) .
$$

If $\Phi \in \mathcal{P}(K)$ and $H \in \mathcal{P}(V \backslash K)$, then the pairing of $\Phi$ and the class $[H]$ of $H$ is given by the integral

$$
\langle\Phi,[H]\rangle=\int_{\partial L}\left\{\Phi \frac{\partial H}{\partial n}-\frac{\partial \Phi}{\partial n} H\right\} d S,
$$

where $L$ is a compact neighborhood with smooth boundary of $K$ in the intersection of $V$ and the domain of $\Phi$.

The Mittag-Leffler theorem for harmonic functions. Let $V_{\lambda}, \lambda \in \Lambda$, be open sets in $\mathbb{R}^{n+1}$. If a family of harmonic functions $H_{\lambda \mu} \in \mathcal{P}\left(V_{\lambda} \cap V_{\mu}\right)$ satisfy

$$
H_{\lambda \mu}+H_{\mu \nu}+H_{\nu \lambda}=0 \quad \text { on } V_{\lambda} \cap V_{\mu} \cap V_{\nu}
$$

for all $V_{\lambda} \cap V_{\mu} \cap V_{\nu} \neq \emptyset$, then there are harmonic functions $H_{\lambda} \in \mathcal{P}\left(V_{\lambda}\right)$ such that

$$
H_{\lambda \mu}=H_{\mu}-H_{\lambda} \quad \text { on } V_{\lambda} \cap V_{\mu} .
$$

In terms of cohomology groups this is equivalent to the statement

$$
H^{1}(V, \mathcal{P})=0 \quad \text { for any open set } V \text { in } \mathbb{R}^{n+1} .
$$

Malgrange [27] proved the theorem as the exactness of

$$
0 \rightarrow \mathcal{P}(V) \rightarrow \mathcal{E}(V) \stackrel{\Delta}{\rightarrow} \mathcal{E}(V) \rightarrow 0,
$$

where $\mathcal{E}$ denotes the sheaf of $C^{\infty}$ functions (cf. Hörmander [14], pp. 12-14).

As in the case of one complex variable, the Mittag-Leffler theorem is proved by the Runge approximation theorem saying that for a compact set $K$ in an open set $V$ in $\mathbb{R}^{n+1}, \mathcal{P}(V)$ is dense in $\mathcal{P}(K)$ if and only if $V \backslash K$ has no relatively compact components in $V$. The Runge theorem can in turn be derived from the Grothendieck theorem.

Proposition 1. $\mathcal{B}(\Omega)$ does not depend on $V$.

Pro of. Suppose that $V \subset W$ are two open sets such that $\widetilde{V}$ and $\widetilde{W}$ are open. We have to prove that the natural mapping

$$
j: \mathcal{P}(W) / \mathcal{P}_{0}(\widetilde{W}) \rightarrow \mathcal{P}(V) / \mathcal{P}_{0}(\widetilde{V})
$$

induced from restriction mappings is an isomorphism.

If $H \in \mathcal{P}(W)$ has a continuous boundary value 0 , then it can be continued to a function in $\mathcal{P}_{0}(\widetilde{W})$ by the reflection principle. Hence $j$ is injective. 
The surjectivity is proved if every $H \in \mathcal{P}(V)$ is decomposed as $H_{1}-H_{0}$ with $H_{1} \in \mathcal{P}(W)$ and $H_{0} \in \mathcal{P}_{0}(\widetilde{V})$. Since $V=W \cap(\widetilde{V} \cup \bar{W})$, it follows from the Mittag-Leffler theorem for two open sets that there are $G_{1} \in \mathcal{P}(W)$ and $G_{0} \in \mathcal{P}(\widetilde{V} \cup \bar{W})$ such that $H=G_{1}-G_{0}$. Then $H_{0}=G_{0}+\check{G}_{0} \in \mathcal{P}_{0}(\widetilde{V})$ and $H_{1}=G_{1}+\check{G}_{0} \in \mathcal{P}(W)$ satisfy the condition, where

$$
\check{G}_{0}(x, t)=-G_{0}(x,-t) .
$$

Definition 2. Let $\Omega_{1} \subset \Omega$ be open sets in $\mathbb{R}^{n}$. Then we choose open sets $V_{1} \subset V$ in $\mathbb{R}_{+}^{n+1}$ such that $\widetilde{V}_{1}=V_{1} \cup \Omega_{1} \cup \bar{V}_{1}$ and $\widetilde{V}=V \cup \Omega \cup \bar{V}$ are open, and define the restriction mapping $\varrho_{\Omega_{1}}: \mathcal{B}(\Omega) \rightarrow \mathcal{B}\left(\Omega_{1}\right)$ by the natural mapping

$$
\mathcal{P}(V) / \mathcal{P}_{0}(\widetilde{V}) \rightarrow \mathcal{P}\left(V_{1}\right) / \mathcal{P}_{0}\left(\widetilde{V}_{1}\right)
$$

induced from restriction mappings.

It follows from Proposition 1 that the mapping $\varrho_{\Omega_{1}}^{\Omega}$ does not depend on $V$ and $V_{1}$.

THEOREM 1. The hyperfunctions $\mathcal{B}(\Omega), \Omega \subset \mathbb{R}^{n}$, with the restriction mappings $\varrho_{\Omega_{1}}^{\Omega}$, form a sheaf.

Proof. In order to prove properties (S.1) and (S.2), let $\Omega_{\lambda}$ and $V_{\lambda}$ be open sets in $\mathbb{R}^{n}$ and $\mathbb{R}_{+}^{n+1}$ respectively such that $\widetilde{V}_{\lambda}=V_{\lambda} \cup \Omega_{\lambda} \cup \bar{V}_{\lambda}$ are open in $\mathbb{R}^{n+1}$. We set $\Omega=\bigcup \Omega_{\lambda}$ and $V=\bigcup V_{\lambda}$. Then $\widetilde{V}=V \cup \Omega \cup \bar{V}$ is also open in $\mathbb{R}^{n+1}$.

To prove (S.1) suppose that $H \in \mathcal{P}(V)$ belongs to $\mathcal{P}_{0}\left(\widetilde{V}_{\lambda}\right)$ for all $\lambda$. Then $H$ belongs to $\mathcal{P}_{0}(\widetilde{V})$ by the reflection principle.

To prove (S.2) suppose that $H_{\lambda} \in \mathcal{P}\left(V_{\lambda}\right)$ satisfy

$$
H_{\lambda \mu}:=H_{\mu}-H_{\lambda} \in \mathcal{P}_{0}\left(\widetilde{V}_{\lambda} \cap \widetilde{V}_{\mu}\right) .
$$

Then it follows from the Mittag-Leffler theorem and the antisymmetrization that there are $F_{\lambda} \in \mathcal{P}_{0}\left(\widetilde{V}_{\lambda}\right)$ such that $H_{\lambda \mu}=F_{\mu}-F_{\lambda}$. Then $H=H_{\lambda}-F_{\lambda}$ is a harmonic function in $\mathcal{P}(V)$ which represents $H_{\lambda}(x,+0)$ on $\Omega_{\lambda}$.

THEOREM 2. The sheaf $\mathcal{B}$ of hyperfunctions over $\mathbb{R}^{n}$ is flabby.

Proof. It is enough to take $V=\mathbb{R}_{+}^{n+1}$ for the representation (2.5).

Definition 3. Let $K$ be a compact set in $\mathbb{R}^{n}$. We define the pairing of $\varphi \in$ $\mathcal{A}(K)$ and $f \in \mathcal{B}_{K}\left(\mathbb{R}^{n}\right)$ as follows. Let $\Phi(x, t) \in \mathcal{P}_{0}(K)$ be the solution of the Cauchy problem

$$
\left\{\begin{array}{l}
\Delta \Phi(x, t)=0, \\
\Phi(x, 0)=0, \\
\partial_{t} \Phi(x, 0)=\varphi(x) .
\end{array}\right.
$$

The Cauchy-Kowalevsky theorem guarantees the existence of a unique solution. We take an $H \in \mathcal{P}_{0}(\widetilde{V} \backslash K)$ such that $f(x)=H(x,+0)$ and define

$$
\langle\varphi, f\rangle:=\int_{S}\left\{\frac{\partial \Phi}{\partial n} H-\Phi \frac{\partial H}{\partial n}\right\} d S
$$


where $S$ is a hypersurface in the intersection of $V$ and the domain of $\Phi$, with boundary in $\Omega \backslash K$, and oriented as a deformation of the natural orientation of $\Omega$ in $\mathbb{R}^{n}$, and $n$ is the upward unit normal on $S$.

THEOREM 3. Under the pairing defined above we have the isomorphism

$$
\mathcal{B}_{K} \cong(\mathcal{A}(K))^{\prime} .
$$

Pr o of. Let $\bar{S}$ be the mirror image of $S$ in (2.15). Then $-S+\bar{S}$ is the boundary of a compact neighborhood $L$ of $K$ in the intersection of $\widetilde{V}$ and the domain of $\Phi$. Since $\Phi$ and $H$ are odd functions in $t$, the right hand side of (2.15) is equal to the same integral over $\bar{S}$ if $n$ is interpreted as the outer unit normal on $\partial L$. The upward normal $n$ in (2.15) is also the outer unit normal on $\partial L$ but the orientation of $S$ is opposite to that of $\partial L$. Therefore the integral (2.15) should be regarded as the integral on the chain $-S$. Hence it is equal to one half of the integral on $\partial L$. It is proved in the same way that

$$
\int_{\partial L}\left\{\frac{\partial \Phi}{\partial n} H-\Phi \frac{\partial H}{\partial n}\right\} d S=0
$$

for any even harmonic function $\Phi$. Hence Grothendieck's duality theorem implies the isomorphism (2.16).

When an analytic functional $T \in(\mathcal{A}(K))^{\prime}$ is given, the corresponding hyperfunction $f(x)$ is represented by the Poisson integral

$$
H(x, t):=\left\langle P(x-y, t), T_{y}\right\rangle,
$$

where

$$
P(x, t):=\frac{2}{\omega_{n+1}} \frac{t}{\left(x^{2}+t^{2}\right)^{(n+1) / 2}}=\frac{1}{(2 \pi)^{n}} \int_{\mathbb{R}^{n}} e^{-t|\xi|^{2}+i x \xi} d \xi
$$

is the Poisson kernel.

In case the dimension $n>1$, Poisson integrals $H(x, t)$ often play an essential role in making detailed study of various classes of (generalized) functions $f(x)$ (e.g. [43]). In this respect our definition of hyperfunctions is in accordance with the tradition of real analysis.

3. Partial differential equations with constant coefficients. Since this is a seminar on partial differential equations, we will show here some applications to linear partial differential equations with constant coefficients, which are modifications, according to our new approach, of the results we reported at the Conference on Generalized Functions held in Katowice in 1966 (cf. [20], [22]).

We define derivatives of hyperfunctions by

$$
\partial_{x}^{\alpha}(H(x,+0))=\left(\partial_{x}^{\alpha} H\right)(x,+0)
$$

Given an $r_{1} \times r_{0}$ system of differential operators

$$
P(\partial): \mathcal{B}^{r_{0}} \rightarrow \mathcal{B}^{r_{1}}
$$


the transposed matrix $P^{\prime}(-\partial)$ may be regarded as the homomorphism $\mathbb{C}[\partial]^{r_{1}} \rightarrow$ $\mathbb{C}[\partial]^{r_{0}}$ of polynomial modules in $\partial$. Then by the Hilbert syzygy theorem there is an exact sequence terminating for some $m \leq n$ :

$$
\begin{aligned}
\mathbb{C}[\partial]^{r_{0}} \stackrel{P^{\prime}(-\partial)}{\longleftarrow} \mathbb{C}[\partial]^{r_{1}} \stackrel{P_{1}^{\prime}(-\partial)}{\longleftarrow} \mathbb{C}[\partial]^{r_{2} \longleftarrow} \ldots \\
\ldots \longleftarrow \mathbb{C}[\partial]^{r_{m-1}} \stackrel{P_{m-1}^{\prime}(-\partial)}{\longleftarrow} \mathbb{C}[\partial]^{r_{m} \longleftarrow} \longleftarrow .
\end{aligned}
$$

Theorem 4. Let $P(\partial)$ and $P_{j}(\partial)$ be as above. Then for any convex open set $\Omega$ in $\mathbb{R}^{n}$ we have the exact sequence

$$
\mathcal{B}(\Omega)^{r_{0}} \stackrel{P(\partial)}{\longrightarrow} \mathcal{B}(\Omega)^{r_{1}} \stackrel{P_{1}(\partial)}{\longrightarrow} \ldots \stackrel{P_{m-1}(\partial)}{\longrightarrow} \mathcal{B}(\Omega)^{r_{m}} \longrightarrow 0 .
$$

In particular, the equation

$$
P(\partial) u=f
$$

has a solution $u \in \mathcal{B}(\Omega)^{r_{0}}$ if and only if the data $f \in \mathcal{B}(\Omega)^{r_{1}}$ satisfies the compatibility condition

$$
P_{1}(\partial) f=0 .
$$

Pro of. Let $V$ be a convex open set in $\mathbb{R}_{+}^{n+1}$ such that $\widetilde{V}=V \cup \Omega \cup \bar{V}$ is convex and open in $\mathbb{R}^{n+1}$. Ehrenpreis [7], [8], Malgrange and Hörmander [14] have proved the theorem with $\mathcal{B}$ replaced by the sheaf $\mathcal{E}$ of infinitely differentiable functions. Hence the last two rows of the following diagram are exact:

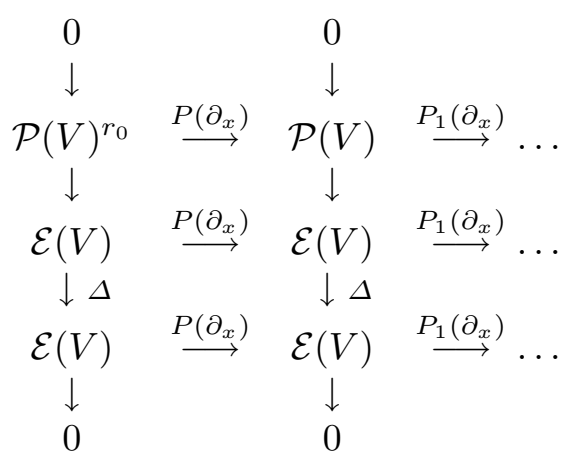

By the Malgrange theorem (2.12) all columns are exact. Thus it follows that the first row is exact.

By the antisymmetrization we have the exact sequence

$$
\mathcal{P}_{0}(\widetilde{V})^{r_{0}} \stackrel{P\left(\partial_{x}\right)}{\longrightarrow} \mathcal{P}_{0}(\widetilde{V})^{r_{1}} \stackrel{P_{1}\left(\partial_{x}\right)}{\longrightarrow} \ldots
$$

Hence the desired exactness follows from the exact sequences

$$
0 \rightarrow \mathcal{P}_{0}(\widetilde{V})^{r_{j}} \longrightarrow \mathcal{P}(V)^{r_{j}} \longrightarrow \mathcal{B}(\Omega)^{r_{j}} \rightarrow 0 .
$$

Similarly we have the following theorem by Harvey [13] and Bengel [1].

TheOREM 5. If $P(\partial)$ is elliptic, then every hyperfunction solution $u$ of $P u=f$ is real-analytic wherever so is $f$. 
In particular, we have the flabby resolutions

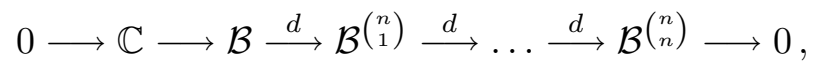

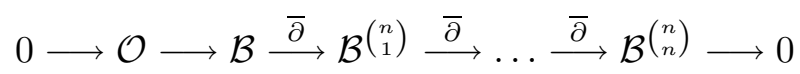

of the constant sheaf $\mathbb{C}$ and the sheaf $\mathcal{O}$ of holomorphic functions over $\mathbb{R}^{n}$ and $\mathbb{C}^{n}$ respectively.

Now it is very easy to prove the basic results employed in the first foundation of the theory of hyperfunctions.

Corollary 1 (Malgrange). We have

$$
\begin{array}{ll}
H^{n}(U, \mathbb{C})=0 & \text { for any open set } U \text { in } \mathbb{R}^{n}, \\
H^{n}(V, \mathcal{O})=0 & \text { for any open set } V \text { in } \mathbb{C}^{n} .
\end{array}
$$

Proof. By definition we have

$$
H^{n}(U, \mathbb{C})=\mathcal{B}(U)^{\left(\begin{array}{l}
n \\
n
\end{array}\right)} / d \mathcal{B}(U)^{\left(\begin{array}{c}
n \\
n-1
\end{array}\right)} .
$$

Given an $n$-form $f \in \mathcal{B}(U)^{\left(\begin{array}{l}n \\ n\end{array}\right)}$, we can extend it to a form $\tilde{f} \in \mathcal{B}\left(\mathbb{R}^{n}\right)^{\left(\begin{array}{l}n \\ n\end{array}\right)}$ by the flabbiness. Theorem 4 asserts the existence of a solution $\widetilde{u} \in \mathcal{B}\left(\mathbb{R}^{n}\right)^{\left(\begin{array}{c}n \\ n-1\end{array}\right)}$ of $d \widetilde{u}=\widetilde{f}$. Its restriction $u$ to $V$ satisfies $d u=f$ on $V$. (3.11) is proved similarly.

Corollary 2 (Köthe-Martineau). If $K$ is a compact set in an open set $V$ in $\mathbb{C}^{n}$ such that

$$
H^{p}(K, \mathcal{O})=0 \quad \text { for } p>0
$$

then we have

$$
H_{K}^{p}(V, \mathcal{O}) \cong \begin{cases}0, & p \neq n \\ \mathcal{O}(K)^{\prime}, & p=n\end{cases}
$$

Pr o of. The local cohomology groups $H_{K}^{p}(V, \mathcal{O})$ are by definition the cohomology groups of the complex

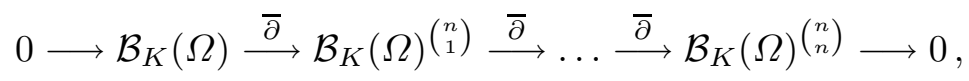

which is dual to the complex

$$
0 \longleftarrow \mathcal{A}(K)^{\left(\begin{array}{l}
n \\
n
\end{array}\right) \stackrel{-\bar{\partial}}{\longleftarrow}} \mathcal{A}(K)^{\left(\begin{array}{c}
n \\
n-1
\end{array}\right)} \stackrel{-\bar{\partial}}{\longleftarrow} \ldots \stackrel{-\bar{\partial}}{\longleftarrow} \mathcal{A}(K) \stackrel{-\bar{\partial}}{\longleftarrow} 0 .
$$

By (3.12), the complex (3.15) is exact except for the 0-th position at which the cohomology group is equal to $\mathcal{O}(K)$. Hence we have (3.13) by the duality of cohomology groups.

Similarly we have the following

COROllary 3 (Alexander-Pontryagin). For any compact set $K$ in an open set $V$ in $\mathbb{R}^{n}$ we have

$$
H_{K}^{p}(V, \mathbb{C}) \cong\left(H^{n-p}(K, \mathbb{C})\right)^{\prime} .
$$

Hence we have a purely analytical proof of the Jordan-Brouwer theorem. 
4. Microlocal analysis. We have so far defined hyperfunctions as boundary values of harmonic functions and have given some applications. All of these could have been done in 1966 before the Conference in Katowice. Actually Bengel [1] had considered boundary values of solutions of elliptic equations to prove his Theorem 5. Sato had also mentioned a definition of hyperfunctions as boundary values of harmonic functions.

It was three years later that microlocal analysis was born, that is, the analysis on the cosphere bundle $S^{*} \Omega=\left(T^{*} \Omega \backslash\{0\}\right) / \mathbb{R}_{+}$or the cotangent bundle $T^{*} \Omega$ over the domain $\Omega$ on which we consider solutions. Whenever there is an epochmaking discovery, there are always forerunners. In this case they are Calderón (1958) and Mizohata (1959) for singular integral operators, Kohn-Nirenberg (1965) and Hörmander (1965) for pseudo-differential operators, and Egorov (1969) and others. The real history started, however, in 1969 at the International Conference on Functional Analysis and Related Topics held in Tokyo. Sato [37] introduced microfunctions as decompositions to the cosphere bundle $S^{*} \Omega$ of singularities of hyperfunctions modulo the real-analytic functions; that is, he defined a sheaf $\mathcal{C}$ over $S^{*} \Omega$ such that

$$
0 \longrightarrow \mathcal{A} \longrightarrow \mathcal{B} \stackrel{\mathrm{sp}}{\longrightarrow} \pi_{*} \mathcal{C} \longrightarrow 0
$$

is exact, where $\pi: S^{*} \Omega \rightarrow \Omega$ is the canonical projection and $\pi_{*} \mathcal{C}$ denotes the direct image so that we have $\mathcal{C}\left(\pi^{-1} \Omega_{1}\right) \cong \mathcal{B}\left(\Omega_{1}\right) / \mathcal{A}\left(\Omega_{1}\right)$ for any open set $\Omega_{1}$ in $\Omega$.

His motivation was to prove Theorem 5 in the variable coefficient case in a natural way. He showed that a linear differential operator $P$ with real-analytic coefficients acts on $\mathcal{C}$ locally in $S^{*} \Omega$, that is, microlocally, and that $P$ is injective at non-characteristic points in $S^{*} \Omega$. Hence we have

$$
\mathrm{SS} u \subset \mathrm{SS}(P u) \cup \operatorname{Char} P,
$$

where SS $u=\operatorname{suppsp} u$. If $P$ is elliptic, i.e., Char $P=\emptyset$, then $u$ is real-analytic wherever so is $P u$.

Hörmander also attended the Conference and conducted a private seminar in which he talked about his idea of Fourier integral operators. His motivation was in Egorov's work on propagation of singularities of solutions of hyperbolic equations.

In 1969-1972 there was a hot competition between Sato's school and Hörmander's. The outcome was monumental papers Hörmander [15] and DuistermaatHörmander [6] on the one hand, and Sato-Kawai-Kashiwara [38] on the other. Each one is 200 pages long or more and contains many results. The highlight of both papers was that they formulated the Huygens principle mathematically and proved it. However, their definitions of fundamental concepts and their proofs of the essentially same results are completely different. Sato et al. employ the cosphere bundle $S^{*} \Omega$, microfunctions $\mathcal{C}$, singularity spectrum SS $u$ and quantized contact transforms whereas Hörmander et al. the cotangent bundle $T^{*} \Omega$, 
wave front sets $W F(u)$ and Fourier integral operators. The analytic wave front set $W F_{A}(u)$ Hörmander introduced later (cf. [16]) coincides with the singularity spectrum SS $u$ for distributions $u$ but the proof was extremely difficult. One reason is that Sato et al. employ higher cohomology groups. For example, the sheaf $\mathcal{C}$ of microfunctions over $S^{*} \mathbb{R}^{n}$ is defined as the $n$th derived cohomology group

$$
\mathcal{C}:=\mathcal{H}_{S^{*} \mathbb{R}^{n}}^{n}\left(\pi^{-1} \mathcal{O}\right)^{a}
$$

with support in $S^{*} \mathbb{R}^{n}$ over the comonoidal transformation $\left(\mathbb{C}^{n} \backslash \mathbb{R}^{n}\right) \cup S^{*} \mathbb{R}^{n}$ with a non-Hausdorff topology. Our motivation is to build a bridge spanning these two schools.

5. Microfunctions as singularities of holomorphic functions. Let $\Omega$ be an open set in $\mathbb{R}^{n}$. We define our disc bundle $D \Omega$ and cosphere bundle $S^{*} \Omega$ by

$$
\begin{gathered}
D \Omega:=\left\{x+i y \in \mathbb{C}^{n} ; x \in \Omega,|y|<1\right\}, \\
S^{*} \Omega:=(\partial D \Omega)^{a}=\{x-i \omega ; x \in \Omega,|\omega|=1\},
\end{gathered}
$$

where $a$ stands for the antipodal mapping.

Note that these are entirely different from Sato's.

Definition 4. The sheaf $\mathcal{C}$ of microfunctions over $\mathbb{R}^{n}$ is defined by the exact sequence of sheaves over $\mathbb{C}^{n}$ :

$$
\left.\left.0 \rightarrow \mathcal{O}\right|_{\left[D \mathbb{R}^{n}\right]} \rightarrow \mathcal{O}\right|_{D \mathbb{R}^{n}} \rightarrow \mathcal{C}^{a} \rightarrow 0
$$

where $\left[D \mathbb{R}^{n}\right]$ denotes the closure $D \mathbb{R}^{n} \cup\left(S^{*} \mathbb{R}^{n}\right)^{a}$ of $D \mathbb{R}^{n}$, and $\left.\mathcal{O}\right|_{A}$ the sheaf which assigns to each open set $W$ in $\mathbb{C}^{n}$ the space

$$
\mathcal{O}(W \cap A)=\lim _{V \supset \vec{W} \cap A} \mathcal{O}(V)
$$

for a locally closed set $A$. $\mathcal{C}^{a}$ means that we evaluate it at the antipodal point. Since the quotient sheaf $\mathcal{C}^{a}$ has the support in the closed set $\left(S^{*} \mathbb{R}^{n}\right)^{a}$, it is naturally regarded as a sheaf over $\left(S^{*} \mathbb{R}^{n}\right)^{a}$.

A microfunction on a neighborhood of a point $(x, \omega) \in S^{*} \mathbb{R}^{n}$ is by the definition a class of holomorphic functions $K(z)$ defined on $V \cap D \mathbb{R}^{n}$ for a neighborhood $V$ of $x-i \omega$ in $\mathbb{C}^{n}$, modulo the holomorphic functions defined on a neighborhood of $x-i \omega$ in $\mathbb{C}^{n}$.

Let $\Sigma$ be an open set in $S^{*} \mathbb{R}^{n}$. If $W$ is an open set in $D \mathbb{R}^{n}$ such that $W \cup \Sigma^{a}$ has a fundamental system of open neighborhoods $\widetilde{W}$ in $\mathbb{C}^{n}$ which is pseudoconvex and contains $\Sigma$ as a closed set, then we have the global representation

$$
\mathcal{C}(\Sigma)=\mathcal{O}(W) / \mathcal{O}\left(W \cup \Sigma^{a}\right) .
$$

In fact, we have the exact sequence of cohomology groups

$$
0 \rightarrow \mathcal{O}\left(W \cup \Sigma^{a}\right) \rightarrow \mathcal{O}(W) \rightarrow \mathcal{C}(\Sigma) \rightarrow H^{1}\left(W \cup \Sigma^{a}, \mathcal{O}\right)=0 .
$$

Since $D \mathbb{R}^{n}$ is strictly pseudoconvex and polynomially convex in $\mathbb{C}^{n}$, we can always find such an open set $W$. Actually the proof of the following theorem shows that we can always take $D \mathbb{R}^{n}$ for $W$. 
Theorem 6 (Kashiwara). The sheaf $\mathcal{C}$ of microfunctions is flabby.

P r o o f. Let $\Sigma$ be an open set in $S^{*} \mathbb{R}^{n}$. It is sufficient to prove that for any open neighborhood $V$ of $D \mathbb{R}^{n} \cup \Sigma^{a}$ in $\mathbb{C}^{n}$ there is a pseudoconvex open neighborhood $\widetilde{W}$ included in $V$.

$W=D \mathbb{R}^{n}$ is strictly pseudoconvex in the sense that it is defined in $\mathbb{C}^{n}$ by the inequality

$$
\varphi(z):=|y|^{2}=\sum_{j=1}^{n} y_{j}^{2}<1
$$

and the Hermitean form

$$
\sum_{j, k=1}^{n} \frac{\partial^{2} \varphi}{\partial z_{j} \partial \overline{z_{k}}} w_{k} \overline{w_{k}}=\frac{1}{2}|w|^{2}
$$

is strictly positive definite. Such a function is called strictly plurisubharmonic.

We take a partition of unity $\sum \chi_{\nu}(z)=1$ on $V \backslash\left(S^{*} \mathbb{R}^{n} \backslash \Sigma\right)^{a}$ and an increasing sequence of compact sets $K_{j}$ in $\Sigma^{a}$ which covers $\Sigma^{a}$. Let $\psi_{j}(z)$ be the sum of all $\chi_{\nu}(z)$ such that $\operatorname{supp} \chi_{\nu} \cap K_{j} \neq \emptyset$. We take an $\varepsilon_{1}>0$ so small that

$$
M_{1}:=\varepsilon_{1} \sup _{\substack{|\alpha|+|\beta| \leq 2 \\ z \in V}}\left|\partial^{\alpha} \bar{\partial}^{\beta} \psi_{1}(z)\right| \leq \frac{1}{4 n^{2}},
$$

and set $M_{-1}=M_{0}=M_{1}$. If $\varepsilon_{1}, \ldots, \varepsilon_{j-1}$ have been chosen, then we set

$$
M_{j}:=\max _{1 \leq k<j}\left\{\varepsilon_{k} \sup _{\substack{|\alpha|+|\beta|=j+1 \\ z \in V}}\left|\partial^{\alpha} \bar{\partial}^{\beta} \psi_{k}(z)\right|\right\}
$$

and choose an $\varepsilon_{j}>0$ so that

$$
\varepsilon_{j} \sup _{|\alpha|+|\beta|=k}\left|\partial^{\alpha} \bar{\partial}^{\beta} \psi_{j}(z)\right| \leq 2^{k-j-2} M_{k-1}, \quad 0 \leq k \leq j+1 .
$$

Then $\psi(z)=\sum_{j=1}^{\infty} \varepsilon_{j} \psi_{j}(z)$ is an infinitely differentiable function such that $\varphi(z)-$ $\psi(z)$ is strictly plurisubharmonic on $\mathbb{C}^{n}$. Since $\psi(z)>0$ on $\Sigma$ and $=0$ outside $V$, it follows that

$$
\widetilde{W}:=\left\{z \in \mathbb{C}^{n} ; \varphi(z)-\psi(z)<0\right\}
$$

is a strictly pseudoconvex open neighborhood of $D \mathbb{R}^{n} \cup \Sigma^{a}$ included in $V$.

The following Grauert theorem is proved in the same way.

Lemma 1. Any open set $\Omega$ in $\mathbb{R}^{n}$ has a fundamental system of pseudoconvex open neighborhoods in $\mathbb{C}^{n}$. In particular, we have

$$
H^{p}(\Omega, \mathcal{A})=0, \quad p>0 .
$$

Hence it follows that if the sheaf $\mathcal{A}$ of real-analytic functions is a subsheaf of a sheaf $\mathcal{F}$ and

$$
0 \rightarrow \mathcal{A} \rightarrow \mathcal{F} \rightarrow \mathcal{G} \rightarrow 0
$$


is an exact sequence of sheaves over $\mathbb{R}^{n}$, then

$$
0 \rightarrow \mathcal{A}(\Omega) \rightarrow \mathcal{F}(\Omega) \rightarrow \mathcal{G}(\Omega) \rightarrow 0
$$

is exact for any open set $\Omega$ in $\mathbb{R}^{n}$.

In order to define the spectral mapping sp $: \mathcal{B} \rightarrow \pi_{*} \mathcal{C}$ which induces the isomorphism

$$
\operatorname{sp}: \mathcal{B}(\Omega) / \mathcal{A}(\Omega) \cong \mathcal{C}\left(\pi^{-1} \Omega\right)
$$

we prepare two lemmas.

Lemma 2 (Kiselman [19], Siciak [41]). Every harmonic function on the ball

$$
B_{R}:=\left\{v=(x, t) \in \mathbb{R}^{n+1} ;|v|<R\right\}
$$

is continued to a holomorphic function on the Lie ball

$$
\widetilde{B_{R}}:=\left\{u=v+i w \in \mathbb{C}^{n+1} ; t(u)<R\right\},
$$

where

$$
\begin{aligned}
t(u) & =\left(|v|^{2}+|w|^{2}+2\left(|v|^{2}|w|^{2}-\langle v, w\rangle^{2}\right)^{1 / 2}\right)^{1 / 2} \\
& =\left(\sum_{j=1}^{n+1}\left|u_{j}\right|^{2}+\left(\left(\sum\left|u_{j}\right|^{2}\right)^{2}-\left|\sum u_{j}^{2}\right|^{2}\right)^{1 / 2}\right)^{1 / 2} .
\end{aligned}
$$

Proof. If $H(v)$ is continuous up to the boundary, then it is represented as the Poisson integral

$$
H(v)=\frac{R^{2}-v^{2}}{\omega_{n+1} R} \int_{|b|=R} \frac{H(b)}{\left((b-v)^{2}\right)^{(n+1) / 2}} d S_{b} .
$$

Hence it is continued analytically to the connected component of $B_{R}$ in $\left\{u \in \mathbb{C}^{n+1} ;(b-u)^{2} \neq 0\right.$ for all $\left.b \in \partial B_{R}\right\}$. The lemma will be proved if we show that the component coincides with the Lie ball $\widetilde{B_{R}}$.

First we note that the boundary of $\widetilde{B_{R}}$ is represented as

$$
\partial \widetilde{B_{R}}=\left\{e^{i \theta}(q+i r) ; \theta \in \mathbb{R}, p, q \in \mathbb{R}^{n+1},|q|+|r|=R,\langle q, r\rangle=0\right\}
$$

(Siciak [41], Lemma 2).

Secondly, we prove that if $u \in \partial \widetilde{B_{R}}$ and $b \in \mathbb{R}^{n+1}$ satisfy $(u-b)^{2}=0$, then $|b| \leq R$.

In view of (5.16) the real part $v$ of $u$ and the imaginary part $w$ are written

$$
v=q \cos \theta-r \sin \theta, \quad w=q \sin \theta+r \cos \theta,
$$

with $q, r \in \mathbb{R}^{n+1}$ satisfying $|q|+|r|=R$ and $\langle q, r\rangle=0$.

The condition $(u-b)^{2}=0$ implies

$$
\begin{aligned}
2\langle q, b\rangle \cos \theta-2\langle r, b\rangle \sin \theta & =\left(q^{2}-r^{2}\right)\left(\cos ^{2} \theta-\sin ^{2} \theta\right)+b^{2}, \\
\langle q, b\rangle \sin \theta+\langle r, b\rangle \cos \theta & =\left(q^{2}-r^{2}\right) \cos \theta \sin \theta .
\end{aligned}
$$


Hence we have, in view of $|q|+|r|=R$,

$$
2\langle q, b\rangle=\cos \theta\left\{2|q| R+\left(b^{2}-R^{2}\right)\right\}, \quad 2\langle r, b\rangle=-\sin \theta\left\{2|r| R+\left(b^{2}-R^{2}\right)\right\} .
$$

If either $q=0$ or $r=0$, then it is easy to see that $|b|=R$. Otherwise, we have the following components of $b$ with respect to the orthogonal unit vectors $q /|q|$ and $r /|r|$ :

$$
\left\langle\frac{q}{|q|}, b\right\rangle=\cos \theta\left\{R+\frac{b^{2}-R^{2}}{2|q|}\right\}, \quad\left\langle\frac{r}{|r|}, b\right\rangle=-\sin \theta\left\{R+\frac{b^{2}-R^{2}}{2|r|}\right\} .
$$

Adding the squares of both sides, we have

$$
\left(b^{2}-R^{2}\right)\left\{\cos ^{2} \theta\left(1-\frac{R}{|q|}-\frac{b^{2}-R^{2}}{4 q^{2}}\right)+\sin ^{2} \theta\left(1-\frac{R}{|r|}-\frac{b^{2}-R^{2}}{4 r^{2}}\right)\right\} \geq 0 .
$$

Since

we have

$$
1-\frac{R}{|q|}-\frac{b^{2}-R^{2}}{4 q^{2}}=\frac{(|q|-|r|)^{2}-b^{2}}{4 q^{2}}
$$

$$
\left(b^{2}-R^{2}\right)\left\{(|q|-|r|)^{2}-b^{2}\right\}\left(\frac{\cos ^{2} \theta}{4 q^{2}}+\frac{\sin ^{2} \theta}{4 r^{2}}\right) \geq 0 .
$$

This implies $(|q|-|r|)^{2} \leq b^{2} \leq R^{2}$.

The proof also shows that for any $u \in \partial \widetilde{B_{R}}$ there is a $b \in \partial B_{R}$ satisfying $(u-b)^{2}=0$. Hence the Lie ball $\widetilde{B_{R}}$ is a component of $\left\{u \in \mathbb{C}^{n+1} ;(b-u)^{2} \neq 0\right.$ for any $\left.b \in \partial B_{R}\right\}$.

The following lemma is a part of the Paley-Wiener theorem for hyperfunctions.

Lemma 3. Let $K$ be a compact convex set in $\mathbb{R}^{n}$. If $f(x) \in \mathcal{B}_{K}\left(\mathbb{R}^{n}\right)$, then its Fourier transform

$$
\widehat{f}(\zeta):=\int e^{-i\langle x, \zeta\rangle} f(x) d x
$$

is an entire function on $\mathbb{C}^{n}$ satisfying the estimates

$$
|f(\zeta)| \leq C_{\varepsilon} \exp \left\{H_{K}(\zeta)+\varepsilon|\zeta|\right\}
$$

for any $\varepsilon>0$ with a constant $C_{\varepsilon}$ depending on $\varepsilon$, where

$$
H_{K}(\zeta)=\sup _{x \in K} \operatorname{Im}\langle x, \zeta\rangle .
$$

Moreover, the Poisson integral of $f$ is represented as

$$
\operatorname{Pf}(x+i y, t)=\frac{1}{(2 \pi)^{n}} \int_{\mathbb{R}^{n}} e^{-t|\xi|+i\langle x+i y, \xi\rangle} \widehat{f}(\xi) d \xi,
$$

and hence is holomorphic on the tube domain $\left\{x+i y \in \mathbb{C}^{n} ;|y|<t\right\}$ for $t>0$.

Pr o of. The space $\mathcal{B}_{K}$ as the strong dual of $\mathcal{A}(K)$ is a Fréchet space, and so is the space $\operatorname{Exp}_{K}$ of all entire functions satisfying (5.18) if we take $C_{\varepsilon}$ as defining semi-norms. 
It is easy to prove that the Fourier transformation $\mathcal{F}: \mathcal{B}_{K} \rightarrow \operatorname{Exp}_{K}$ and the integral $I: \operatorname{Exp}_{K} \rightarrow \mathcal{P}_{0}\left(\mathbb{R}^{n+1} \backslash K\right)$ defined by (5.20) are continuous linear mappings. If $f(x)=\delta(x-y), y \in K$, then $(I \circ \mathcal{F}) f$ is clearly equal to the Poisson integral $P f$. The linear combinations of such $\delta(x-y)$ are dense in $\mathcal{B}_{K}$ and the Poisson integral $P: \mathcal{B}_{K} \rightarrow \mathcal{P}_{0}\left(\mathbb{R}^{n+1} \backslash K\right)$ is continuous. Hence we have the identity (5.20) for all $f \in \mathcal{B}_{K}$.

Definition 5. Let $f \in \mathcal{B}(\Omega)$ be a hyperfunction on an open set $\Omega$ in $\mathbb{R}^{n}$. We choose an upper neighborhood $V \subset \mathbb{R}_{+}^{n+1}$ of $\Omega$ so large that every closed ball of radius 1 in $\mathbb{R}_{+}^{n+1}$ touching an $x \in \Omega$ is entirely in $V$ except for $x$, and we take a harmonic function $H \in \mathcal{P}(V)$ such that $f(x)=H(x,+0)$. Then it follows from Lemmas 1 and 2 that $H(x, 1)$ has an analytic continuation $H(x+i y, 1)$ to $\pi^{-1} \Omega$ and its class in $\mathcal{C}^{a}\left(\pi^{-1} \Omega\right)$ does not depend on the choice of the defining harmonic function $H(x, t)$, which we define to be sp $f$ :

$$
(\operatorname{sp} f)_{(x, \omega)}:=[H(x+i y, 1)]_{y=-\omega} .
$$

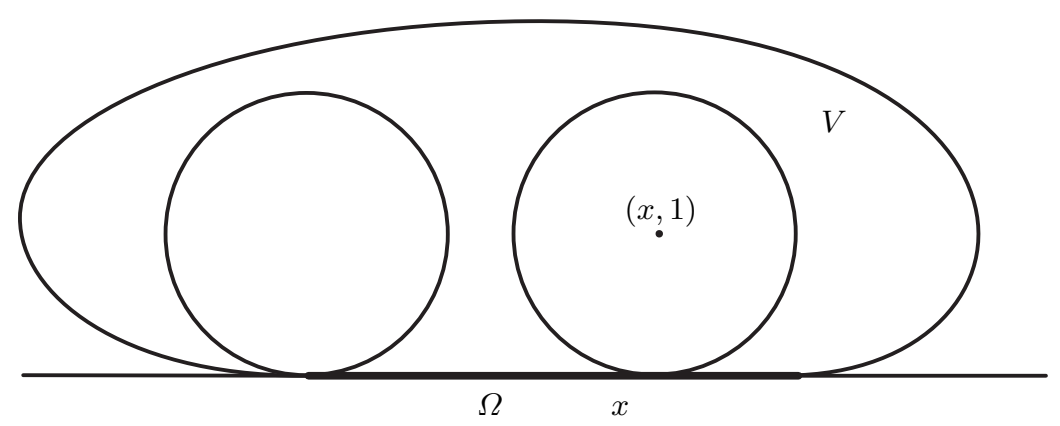

Fig. 2

THEOREM 7. The spectral mapping sp induces the isomorphism

$$
\mathcal{B}(\Omega) / \mathcal{A}(\Omega) \stackrel{\sim}{\rightarrow} \mathcal{C}\left(\pi^{-1} \Omega\right)
$$

for any open set $\Omega$ in $\mathbb{R}^{n}$.

Proof. As we remarked after Lemma 1 this is equivalent to the exactness of the sequence (4.1) of sheaves. Therefore it is sufficient to prove (5.22) for any relatively compact open set $\Omega$ in $\mathbb{R}^{n}$.

To prove the surjectivity, let $[K(x+i y)]$ be a class in $\mathcal{O}\left(D \mathbb{R}^{n}\right) / \mathcal{O}\left(D \mathbb{R}^{n} \cup\right.$ $\left.S^{*} \Omega^{a}\right) \cong \mathcal{C}\left(\pi^{-1} \Omega\right)$. We have to find an $H(x, t) \in \mathcal{P}(V)$, for a sufficiently large upper neighborhood $V$ of $\Omega$, such that

$$
K(x+i y)-H(x+i y, 1) \in \mathcal{O}\left(S^{*} \Omega^{a}\right) .
$$

We choose a relatively compact smooth domain $\Omega_{0}$ in $\mathbb{R}^{n}$ such that all balls of radius $\sqrt{2}$ with center in $\Omega$ are included in $\Omega_{0}$, and define for $t>1$

$$
H(x, t):=\int_{\Omega_{0}} P(x-w, t-1) K(w) d w
$$


where $P(x, t)$ is the Poisson kernel. This is originally a harmonic function on the upper half space $t>1$ and has an analytic continuation to $\{(x+i y, t) ; t>$ $\left.1, x \in \mathbb{R}^{n},|y|<t-1\right\}$. Suppose $t>2$. Then, since the kernel $P(x+i y, t-1)$ is holomorphic on the domain $\left\{x+i y \in \mathbb{C}^{n} ; x^{2}-y^{2}+(t-1)^{2}>0\right\}$, we can deform the integral domain $\Omega_{0}$ into

$$
\Omega_{s}(\omega):=\left\{u-i \omega s \min \left\{1, \operatorname{dis}\left(u, \partial \Omega_{0}\right)\right\} ; u \in \Omega_{0}\right\}
$$

for $\omega \in S^{n-1}$ and $0 \leq s<1$. Then the integral represents a holomorphic function on $\left\{(z, t) ; t>1, \operatorname{Re}(z-w)^{2}+(t-1)^{2}>0\right.$ for all $\left.w \in \Omega_{s}(\omega)\right\}$. Hence $H(x, t)$ has an analytic continuation $H(z, t)$ to the domain $\{(x+i y, t) ; t>1, x \in$ $\left.\Omega_{0},|y|<\min \left\{t-1,\left((t-1)^{2}+\operatorname{dis}^{2}\left(x, \partial \Omega_{0}\right)\right)^{1 / 2}\right\}\right\}$. The derivative $\partial_{t} H(z, t)$ is also holomorphic there.

Since $H(x+i y, t)$ satisfies the wave equation

$$
\left(\partial_{t}^{2}-\Delta_{y}\right) H(x+i y, t)=0
$$

as a function of $y$ and $t$, it can be continued to a holomorphic function on a neighborhood of $V_{0}=\left\{(x, t) ; x \in \Omega_{0}, t>1-\min \left\{1, \operatorname{dis}\left(x, \partial \Omega_{0}\right)\right\}\right\}$ with the aid of the fundamental solution of the wave equation.

Thus $H(x, t)$ is continued to a harmonic function on the upper neighborhood $V=V_{0} \cup\left\{(x, t) ; x \in \mathbb{R}^{n}, t>1\right\}$ of $\Omega$, and we have by (5.24)

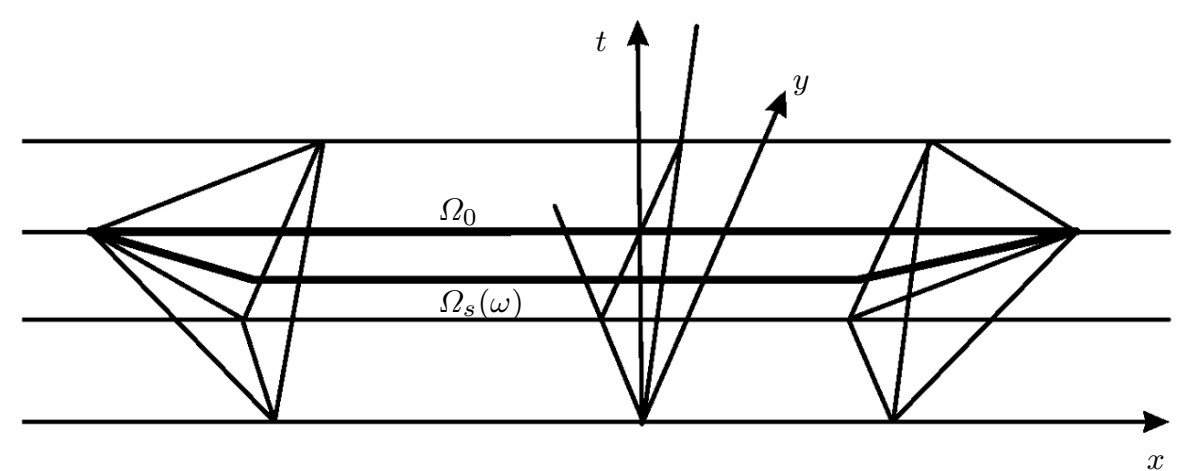

Fig. 3

If $f(x)$ is a real-analytic function on a compact domain $\Omega_{0}$, then the same proof shows that its Poisson integral

$$
H(x, t):=\int_{\Omega_{0}} P(x-y, t) f(t) d y
$$

has an analytic continuation $H(x+i y, 1) \in \mathcal{O}\left(D \Omega \cup S^{*} \Omega^{a}\right)$ for the interior $\Omega$ of $\Omega_{0}$.

Finally, suppose that an $H(x, t) \in \mathcal{P}\left(\mathbb{R}_{+}^{n+1}\right)$ has the analytic continuation $H(x+i y, 1) \in \mathcal{O}\left(D \Omega \cup S^{*} \Omega^{a}\right)$ for a relatively compact domain $\Omega$. Then we can 
construct as above a harmonic function $K(x, t)$ on $\Omega \times\{t \geq 0\}$ such that

$$
K(x+i y, 1)=H(x+i y, 1), \quad x+i y \in D \Omega .
$$

Hence it follows from the reflection principle that

$$
H(x, t)=K(x, t)+K(x, 2-t)-H(x, 2-t) .
$$

The right hand side being real-analytic up to $t=0, H(x,+0)$ is real-analytic on $\Omega$.

6. Boundary values of holomorphic functions on tuboids. Let $\Omega$ be an open set in $\mathbb{R}^{n}$, and $\Gamma$ a convex open cone in $\mathbb{R}^{n} \backslash\{0\}$. An open set $W$ in $\mathbb{C}^{n}$ is said to be a tuboid of profile $\Omega+i \Gamma$ if $W \subset \Omega+i \Gamma$, and if for any open subset $\Omega_{0} \Subset \Omega$ and subcone $\Gamma_{0} \Subset \Gamma$ (i.e. $\Gamma_{0} \cap S^{n-1} \Subset \Gamma \cap S^{n-1}$ ) there is an $r>0$ such that

$$
\Omega_{0}+i\left\{y \in \Gamma_{0} ;|y|<r\right\} \subset W .
$$

For any holomorphic function $F(z) \in \mathcal{O}(W)$ we define its boundary value $F(x+$ $i \Gamma 0) \in \mathcal{B}(\Omega)$ as follows.

Let $\Omega_{0}$ and $\Gamma_{0}$ be as above. We take a small $\gamma \in \Gamma_{0}$ and define

$$
H_{\gamma}(z, t):=\int_{\Omega_{0}+i \gamma} P(z-w, t) F(w) d w .
$$

This is originally a harmonic function on $\left\{(x+i y, t) ; t>0, x \in \mathbb{R}^{n},|y-\gamma|<t\right\}$. If $t>2|\gamma|$ and $|y-\gamma| \leq|\gamma|$, then the integral chain is deformed into

$$
\Omega_{s}:=\left\{x+i \gamma\left(1-s \max \left\{1, \operatorname{dis}\left(x, \partial \Omega_{0}\right) /|\gamma|\right\}\right) ; x \in \Omega_{0}\right\}
$$

for $0 \leq s<1$. Therefore if

$$
\Omega_{\gamma}:=\left\{x \in \Omega_{0} ; d\left(x, \partial \Omega_{0}\right)>|\gamma|\right\}
$$

is not empty, $H_{\gamma}(z, t)$ is continued to a harmonic function on $\Omega_{\gamma} \times\{t>0\}$.

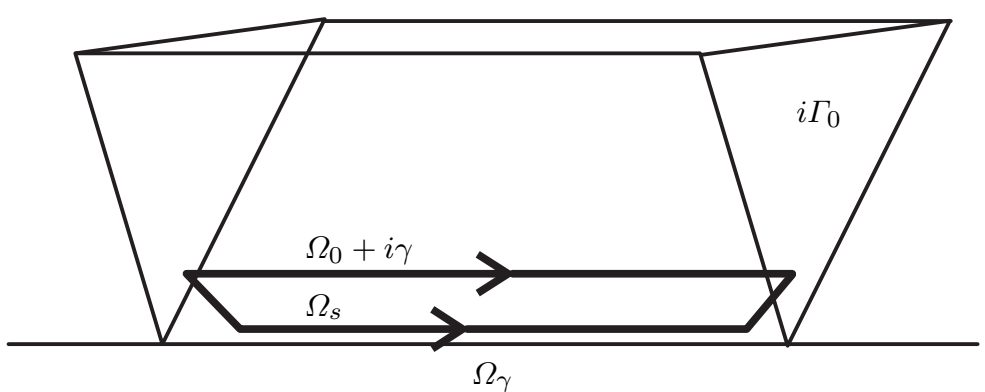

Fig. 4

Definition 6. For a holomorphic function $F(x+i y) \in \mathcal{O}(W)$ on a tuboid of profile $\Omega+i \Gamma$, its boundary value on $\Omega_{\gamma}$ is defined by

$$
F(x+i \Gamma 0):=H_{\gamma}(x,+0), \quad x \in \Omega_{\gamma} .
$$


It is easily proved that $F(x+i \Gamma 0)$ does not depend on $\gamma$ and $\Omega_{0}$ on the common domain of definition. Hence the boundary value $F(x+i \Gamma 0)$ is actually defined on $\Omega$.

We write $F(z) \in \mathcal{O}(\Omega+i \Gamma 0)$ if it is a holomorphic function on a tuboid $W$ of profile $\Omega+i \Gamma$. Thus the boundary value is a linear mapping $\mathcal{O}(\Omega+i \Gamma 0) \rightarrow \mathcal{B}(\Omega)$.

TheOREM 8. Let $\Gamma$ be a convex open cone in $\mathbb{R}^{n} \backslash\{0\}$. A hyperfunction $f(x) \in$ $\mathcal{B}(\Omega)$ on an open set $\Omega$ in $\mathbb{R}^{n}$ has its singularity spectrum

$$
\operatorname{suppsp} f \subset \Omega \times\left(\Gamma^{\circ} \cap S^{n-1}\right)
$$

if and only if it is the boundary value $F(x+i \Gamma 0)$ of a holomorphic function $F(z)$ on a tuboid of profile $\Omega+i \Gamma$. Here $\Gamma^{\circ}$ denotes the polar of $\Gamma$ :

$$
\Gamma^{\circ}:=\left\{\xi \in \mathbb{R}^{n} ;\langle y, \xi\rangle \geq 0 \text { for all } y \in \Gamma\right\} .
$$

The correspondence between $F(z) \in \mathcal{O}(\Omega+i \Gamma 0)$ and $F(x+i \Gamma 0) \in \mathcal{B}(\Omega)$ is oneto-one. If $F(x+i \Gamma 0)$ vanishes on an open subset, it vanishes on its connected component.

Pro of. Suppose that $F(z) \in \mathcal{O}(\Omega+i \Gamma 0)$. Then $H_{\gamma}(x+i y, t)$ constructed above is holomorphic on $\Omega_{0}+i\{\gamma+y ;|y|<t\}$. Since $\gamma$ can be an arbitrary small vector in $\Gamma, H_{\gamma}(x+i y, 1)$ is holomorphic on $\left(S^{*} \Omega \backslash\left(\Omega+i \Gamma^{\circ}\right)\right)^{a}$.

Here we note that if $F\left(x+i \Gamma_{1} 0\right)=0$ on an open subset $\Omega_{1}$ of $\Omega$, then $F(z)$ vanishes identically on the connected component of $\Omega_{1}+i\left\{y \in \Gamma_{1} ;|y|<r\right\}$. In fact, then $H_{\gamma}(x, t)$ is analytic on a neighborhood of $\Omega_{\gamma} \times\{t=0\}$ and $H_{\gamma}(x, 0)=0$ for $x \in \Omega_{\gamma}$. On the other hand, we have

$$
H_{\gamma}(x+i y, 0)=F(x+i y), \quad x \in \Omega_{1}, y \in \Gamma_{2},|y|<|\gamma| / 2
$$

for an open cone $\Gamma_{2} \subset \Gamma_{1}$ by deforming the integral chain of (6.1) as in the proof of Theorem 7 . Hence $F(z)$ is analytically continued to $\Omega_{\gamma}$ and vanishes there. Then, the unique continuation theorem for holomorphic functions implies $F(z) \equiv 0$.

In particular, the boundary value mapping $\mathcal{O}(\Omega+i \Gamma 0) \rightarrow \mathcal{B}(\Omega)$ is injective, and the boundary value $F(x+i \Gamma 0)$ has the unique continuation property in $x$.

Conversely, suppose that (6.3) holds. Then the harmonic function $K(x, t)$ constructed for the inverse image of the spectral mapping is continued to the holomorhic function $K(x+i y, 0)$ on $\Omega_{1}+i\left\{y \in \Gamma_{1} ;|y|<r\right\}$ for any $\Omega_{1} \Subset \Omega$ and $\Gamma_{1} \Subset \Gamma$ with a constant $r>0$. It is easy to see that $K\left(x+i \Gamma_{1} 0\right)=K(x,+0)$ on $\Omega_{1}$. Since $f(x)-K(x,+0)$ is real-analytic, there is an $F(z) \in \mathcal{O}\left(\Omega_{1}+i \Gamma_{1} 0\right)$ such that $f(x)=F(x+i \Gamma 0)$ on $\Omega_{1}$.

As we remarked above, $F(z)$ is uniquely determined by $f(x)$. Hence $F(z)$ is defined on a tuboid of profile $\Omega+i \Gamma$.

The flabbiness of $\mathcal{C}$ (Theorem 6 ), the exact sequence

$$
0 \rightarrow \mathcal{A} \rightarrow \mathcal{B} \rightarrow \pi_{*} \mathcal{C} \rightarrow 0
$$

(Theorem 7) and the characterization of microfunctions with support in $K \times\left(\Gamma^{\circ} \cap\right.$ $S^{n-1}$ ) (Theorem 8) characterize the sheaf $\mathcal{C}$ over $S^{*} \mathbb{R}^{n}$ up to an isomorphism. 
Therefore, our microfunctions are isomorphic to Sato's.

The support supp sp $f$ as a microfunction is called the singularity spectrum of the hyperfunction $f$, and is denoted by $\operatorname{SS} f$.

The following theorem is called Martineau's edge of the wedge theorem [31].

Theorem 9. Let $\Gamma_{j}, j=1, \ldots, m$, be convex open cones in $\mathbb{R}^{n} \backslash\{0\}$, and set

$$
\Gamma_{j k}:=\Gamma_{j}+\Gamma_{k} .
$$

Then we have for any open set $\Omega$ in $\mathbb{R}^{n}$ the isomorphism

$$
\left\{f \in \mathcal{B}(\Omega) ; \operatorname{SS} f \subset \Omega \times\left(\bigcup_{j=1}^{m} \Gamma_{j}^{\circ} \cap S^{n-1}\right)\right\} \cong \bigoplus_{j=1}^{m} \mathcal{O}\left(\Omega+i \Gamma_{j} 0\right) / \sim,
$$

where $\left(F_{j}(z) \in \mathcal{O}\left(\Omega+i \Gamma_{j} 0\right)\right) \sim 0$ if and only if there are $F_{j k}(z) \in \mathcal{O}\left(\Omega+\Gamma_{j k} 0\right)$ such that

$$
\begin{gathered}
F_{j k}(z)+F_{k j}(z)=0, \\
F_{j}(z)=\sum_{k=1}^{m} F_{j k}(z) .
\end{gathered}
$$

Here $\Gamma_{j k}$ are convex open cones but they are not necessarily in $\mathbb{R}^{n} \backslash\{0\}$. In that case $\mathcal{O}\left(\Omega+i \Gamma_{j k} 0\right)$ denotes the real-analytic functions on $\Omega$.

P r o of. We consider the linear mapping

$$
\mathrm{bd}: \bigoplus \mathcal{O}\left(\Omega+i \Gamma_{j} 0\right) \rightarrow \mathcal{B}(\Omega)
$$

which sends $\left(F_{j}(z)\right)$ into $\sum F_{j}\left(x+i \Gamma_{j} 0\right)$. Its image is clearly in the left hand side of (6.6). If $f(x) \in \mathcal{B}(\Omega)$ has its singularity spectrum in $\Omega \times \bigcup \Gamma_{j}^{\circ}$, then by the flabbiness of the microfunctions, $\operatorname{sp} f$ is decomposed into the sum $\sum \operatorname{sp} f_{j}$ with SS $f_{j} \subset \Omega \times \Gamma_{j}^{\circ}$. Each $f_{j}$ is written the boundary value $F_{j}\left(x+i \Gamma_{j} 0\right)$ of an $F_{j}(z) \in \mathcal{O}\left(\Omega+i \Gamma_{j} 0\right)$. Since $f-\sum f_{j}$ is real-analytic, it may be added to one of $f_{j}$.

Suppose that $F_{j}(z) \in \mathcal{O}\left(\Omega+i \Gamma_{j} 0\right)$ satisfy $\sum_{j=1}^{m} F_{j}\left(x+i \Gamma_{j} 0\right)=0$ in $\mathcal{B}(\Omega)$. If $m=1$, then we have $F_{1}(z) \equiv 0$ by Theorem 8 . In general, SS $F_{m}\left(x+i \Gamma_{m} 0\right)$ is included in $\Omega \times\left(\Gamma_{m}^{\circ} \cap \bigcup_{j=1}^{m-1} \Gamma_{j}^{\circ}\right)$. Since $\Gamma_{m j}^{\circ}=\Gamma_{m}^{\circ} \cap \Gamma_{j}^{\circ}$ cover $\Gamma_{m}^{\circ} \cap \bigcup_{j=1}^{m-1} \Gamma_{j}^{\circ}$, there are $F_{m j}(z) \in \mathcal{O}\left(\Omega+i \Gamma_{m j} 0\right)$ such that $F_{m}(z)=\sum_{j=1}^{m-1} F_{m j}(z)$. Let $F_{j m}(z)=$ $-F_{m j}(z)$ and subtract it from $F_{j}(z)$. Then we have the same situation with $m-1$ functions.

The converse is trivial.

If $\left\{\Gamma_{j}^{\circ}\right\}$ covers $\mathbb{R}^{n}$, we have a representation of all hyperfunctions on $\Omega$ as the sum of the boundary values of holomorphic functions on tuboids of profile $\Omega+i \Gamma_{j} 0$. Kaneko [17] adopted this as the definition of hyperfunctions.

7. Hyperfunctions and microfunctions with a prescribed singularity or regularity. Applications. We have so far considered hyperfunctions and 
associated microfunctions. There are, however, infinitely many classes of generalized functions as well as regular functions between the hyperfunctions and the real-analytic functions. An advantage of our approach to hyperfunctions and microfunctions is that it is then very easy to characterize those classes of generalized functions or regular functions among the hyperfunctions and hence to introduce the corresponding classes of microfunctions.

For the sake of brevity we consider here only Schwartz' distributions $\mathcal{D}^{\prime}$, the ultradistributions $\mathcal{D}^{(s) \prime}$ and $\mathcal{D}^{\{s\} '}$ of Gevrey classes and the associated classes $\mathcal{E}, \mathcal{E}^{(s)}$ and $\mathcal{E}^{\{s\}}$ of regular functions. We denote by $*$ the empty symbol or $(s)$ or $\{s\}$ for $1<s<\infty . \mathcal{E}^{*}(\Omega)$ stands for the class of infinitely differentiable functions $f(x)$ on $\Omega$ such that for any compact set $K$ in $\Omega$

$$
\sup _{x \in K}\left|\partial^{\alpha} f(x)\right| \leq\left\{\begin{array}{l}
C_{\alpha}, \text { with a constant } C_{\alpha} \text { if } *=\emptyset \\
C h^{|\alpha|}|\alpha| !^{s}, \text { for any } h>0 \text { with a constant } C \text { if } *=(s), \\
C h^{|\alpha|}|\alpha| !^{s}, \text { with constants } h \text { and } C \text { if } *=\{s\} .
\end{array}\right.
$$

Clearly $\mathcal{E}^{*}(\Omega), \Omega \subset \mathbb{R}^{n}$, form a sheaf. $\mathcal{D}^{*}(\Omega)$ denotes its subclass of all functions with compact support in $\Omega$. The space $\mathcal{D}^{*}(\Omega)$ has a natural locally convex topology and its dual $\mathcal{D}^{* \prime}(\Omega)$ is defined to be the space of (ultra-)distributions of class *. If $\Omega_{1} \subset \Omega$, then the restriction mapping $\varrho_{\Omega_{1}}: \mathcal{D}^{* \prime}(\Omega) \rightarrow \mathcal{D}^{* \prime}\left(\Omega_{1}\right)$ is defined naturally, and $\mathcal{D}^{* \prime}(\Omega), \Omega \subset \mathbb{R}^{n}$, form a sheaf. We have natural inclusion relations:

$$
\begin{aligned}
\mathcal{A} \subset \mathcal{E}^{(s)} \subset \mathcal{E}^{\{s\}} \subset \mathcal{E}^{(t)} \subset \mathcal{E} \subset \mathcal{D}^{\prime} \subset \mathcal{D}^{(t) \prime} \subset \mathcal{D}^{\{s\} \prime} \subset \mathcal{D}^{(s) \prime} \subset \mathcal{B}, \\
1<s<t<\infty .
\end{aligned}
$$

A growth function $G(t)$ of class $*$ is defined by

$$
G(t)=\left\{\begin{array}{l}
-h \log t \quad \text { for some } h>0 \text { if } *=\emptyset, \\
\sup _{p} \log \frac{1}{t^{p} H_{p}(p !)^{s-1}}
\end{array}\right.
$$

where

$$
H_{p}:= \begin{cases}h^{p} & \text { for some } h>0 \text { if } *=(s), \\ h_{1} \ldots h_{p} W W & \text { for some sequence } h_{p} \nearrow \infty \text { if } *=\{s\} .\end{cases}
$$

Note that $G(t)$ is equivalent to $(h t)^{-1 /(s-1)}$ if $*=(s)$.

Theorem 10. Let $\Omega \subset \mathbb{R}^{n}$ and $V \subset \mathbb{R}_{+}^{n+1}$ be as in Definition 1 . Then the following conditions are equivalent for $H(x, t) \in \mathcal{P}(V)$ :

(a) $H(x,+0) \in \mathcal{D}^{* \prime}(\Omega)$;

(b) $H(x, t)$ converges in $\mathcal{D}^{* \prime}(\Omega)$ as $t \rightarrow 0$;

(c) For any $K \Subset \Omega$ there are a growth function $G(t)$ of class $*$ and constants $C$ and $\varepsilon>0$ such that

$$
\sup _{x \in K}|H(x, t)| \leq C \exp G(t), \quad 0<t<\varepsilon .
$$

Under each of these conditions, $H(x, t)$ converges to $H(x,+0)$ in $\mathcal{D}^{* \prime}(\Omega)$. 
An (ultra-)differential operator $P(\partial)$ of class $*$ is defined by

$$
P(\partial)=\sum_{|\alpha|=0}^{\infty} a_{\alpha} \partial^{\alpha},
$$

where $a_{\alpha} \in \mathbb{C}$, and the $a_{\alpha}$ vanish for sufficiently large $|\alpha|$ if $*=\emptyset$;

$$
\left|a_{\alpha}\right| \leq C k^{|\alpha|} /(|\alpha| !)^{s}
$$

with constants $k$ and $C$ if $*=(s)$; and (7.7) holds for any $k>0$ with a constant $C$ if $*=\{s\}$.

THEOREM 11. Under the same assumptions on $\Omega$ and $V$ the following conditions are equivalent for $H(x, t) \in \mathcal{P}(V)$ :

(a) $H(x,+0) \in \mathcal{E}^{*}(\Omega)$;

(b) $H(x, t)$ converges in $\mathcal{E}^{*}(\Omega)$ as $t \rightarrow 0$;

(c) For any differential operator $P(\partial)$ of class $*, P\left(\partial_{x}\right) H(x, t)$ is locally bounded in a neighborhood of $\Omega$.

Under each of these conditions, $H(x, t)$ converges to $H(x,+0)$ in $\mathcal{E}^{*}(\Omega)$.

A proof of Theorems 10 and 11 is given in [24]. Similarly we have the following

THEOREM 12. The following conditions are equivalent for a holomorphic function $F(z)$ on a tuboid of profile $\Omega+i \Gamma$ :

(a) The boundary value $F(x+i \Gamma 0)$ in the sense of hyperfunctions belongs to $\mathcal{D}^{* \prime}(\Omega)\left(\right.$ resp. $\left.\mathcal{E}^{*}(\Omega)\right)$;

(b) $F(x+i y)$ converges in $\mathcal{D}^{* \prime}(\Omega)\left(\right.$ resp. $\left.\mathcal{E}^{*}(\Omega)\right)$ as y tends to zero in some (and any) closed cone $\Gamma_{1} \Subset \Gamma$;

(c) For any compact set $K$ in $\Omega$ and closed cone $\Gamma_{1} \Subset \Gamma$ there are a growth function $G(t)$ of class $*$ and constants $C$ and $r$ such that

$$
\sup _{x \in K}|F(x+i y)| \leq C \exp G(|y|), \quad y \in \Gamma_{1},|y|<r
$$

(resp. $P\left(\partial_{z}\right) F(z)$ is bounded on $K+i\left\{y \in \Gamma_{1} ;|y|<r\right\}$ for any ultradifferential operator $P(\partial)$ of class $*)$.

Under each of these conditions, the topological limit coincides with $F(x+i \Gamma 0)$.

From now on we write $*=(\infty)$ instead of $*=\emptyset$ to avoid confusion.

Definition 7 . We denote by $\left.\mathcal{O}^{*}\right|_{D \mathbb{R}^{n}}\left(\right.$ resp. $\left.\left.\mathcal{O}_{*}\right|_{D \mathbb{R}^{n}}\right)$ the sheaf over $\mathbb{C}^{n}$ of holomorphic functions on $D \mathbb{R}^{n}$ with the growth condition of class $*$ (resp. with the regularity condition of class $*$ ), and define the sheaf $\mathcal{C}^{*}$ of microfunctions of class $*$ (resp. the sheaf $\mathcal{C}^{*}$ of microfunctions of regular class $*$ ) by the exact sequence

$$
\begin{gathered}
\left.\left.0 \rightarrow \mathcal{O}\right|_{\left[D \mathbb{R}^{n}\right]} \rightarrow \mathcal{O}^{*}\right|_{D \mathbb{R}^{n}} \rightarrow\left(\mathcal{C}^{*}\right)^{a} \rightarrow 0 \\
\left(\text { resp. }\left.\left.0 \rightarrow \mathcal{O}\right|_{\left[D \mathbb{R}^{n}\right]} \rightarrow \mathcal{O}_{*}\right|_{D \mathbb{R}^{n}} \rightarrow\left(\mathcal{C}_{*}\right)^{a} \rightarrow 0\right) .
\end{gathered}
$$


Then we have the exact sequences

$$
\begin{gathered}
0 \rightarrow \mathcal{A} \rightarrow \mathcal{D}^{* \prime} \stackrel{\mathrm{sp}}{\rightarrow} \pi_{*} \mathcal{C}^{*} \rightarrow 0, \\
0 \rightarrow \mathcal{A} \rightarrow \mathcal{E}^{*} \stackrel{\mathrm{sp}}{\rightarrow} \pi_{*} \mathcal{C}_{*} \rightarrow 0 .
\end{gathered}
$$

The sheaves $\mathcal{C}^{*}$ and $\mathcal{C}_{*}$ are not flabby but have a little weaker property of suppleness. Hence we have Martineau's edge of the wedge theorem for $\mathcal{D}^{* \prime}$ and $\mathcal{E}^{*}$.

The microfunctions $\mathcal{C}^{(\infty)}$ and $\mathcal{C}_{(\infty)}$ corresponding to the distributions $\mathcal{D}^{\prime}$ and the infinitely differentiable functions $\mathcal{E}$ have been introduced by Bony [3] and Bengel-Schapira [2]. De Roever [5] and Eida [9] extended them to the microfunctions $\mathcal{C}^{*}$ and $\mathcal{C}_{*}$ corresponding to the ultradistributions and the ultradifferentiable functions of general class $*$. Bony [3] employed $\mathcal{C}^{(\infty)}$ to prove the propagation of analytic singularities along bicharacteristic manifolds of distribution solutions of a class of microdifferential equations satisfying the Levi condition.

Eida [9] has proved that SKK's reduction to canonical forms of microdifferential operators with characteristics of constant multiplicity is realized in Gevrey class $*$ under the irregularity condition $* \leq \sigma /(\sigma-1)$ (where the irregularity $\sigma$ is defined in [23]), and thus has established the invariance of support of $\mathcal{C}^{*}$ - and $\mathcal{C}_{*}$-solutions under the bicharacteristic flows. A sketch of proof is given in [24].

\section{References}

[1] G. Bengel, Das Weylsche Lemma in der Theorie der Hyperfunktionen, Math. Z. 96 (1967), 373-392.

[2] G. Bengel et P. Schapira, Décomposition microlocale analytique des distributions, Ann. Inst. Fourier (Grenoble) 29 (3) (1979), 101-124.

[3] J.-M. Bony, Propagation des singularités différentiables pour une classe d'opérateurs différentiels à coefficients analytiques, Astérisque 34-35 (1976), 43-91.

[4] J.-M. Bony et P. Schapira, Propagation des singularités analytiques pour les solutions des équations aux dérivées partielles, Ann. Inst. Fourier (Grenoble) 26 (1) (1976), 81-140.

[5] J. W. de Roever, Hyperfunctional singular support of ultradistributions, J. Fac. Sci. Univ. Tokyo Sect. IA Math. 31 (1984), 585-631.

[6] J. J. Duistermaat and L. Hörmander, Fourier integral operators. II, Acta Math. 128 (1972), 183-269.

[7] L. Ehrenpreis, A fundamental principle for systems of linear differential equations with constant coefficients and some of its applications, in: Proc. Internat. Symp. on Linear Spaces, 1961, Jerusalem, 161-174.

[8] - Fourier Analysis in Several Complex Variables, Wiley-Interscience, New York 1970.

[9] A. Eida, On microlocal decomposition of ultradistributions, Master's Thesis, Univ. Tokyo, 1989 (in Japanese).

[10] H. Grauert, On Levi's problem and the imbedding of real-analytic manifolds, Ann. of Math. 68 (1958), 460-472.

[11] A. Grothendieck, Sur les espaces de solutions d'une classe générale d'équations aux dérivées partielles, J. Analyse Math. 2 (1952-53), 243-280.

[12] _ - Local Cohomology, Lecture Notes in Math. 41, Springer, Berlin 1967. 
[13] R. Harvey, Hyperfunctions and linear partial differential equations, Proc. Nat. Acad. Sci. U.S.A. 55 (1966), 1042-1046.

[14] L. Hörmander, An Introduction to Complex Analysis in Several Variables, Van Nostrand, Princeton 1966.

[15] -, Fourier integral operators. I, Acta Math. 127 (1971), 79-183.

[16] - , The Analysis of Linear Partial Differential Operators I, Springer, Berlin 1983.

[17] A. Kaneko, Introduction to Hyperfunctions, Kluwer, Dordrecht 1988.

[18] M. Kashiwara, T. Kawai and T. Kimura, Foundations of Algebraic Analysis, Princeton Univ. Press, Princeton 1986.

[19] C. O. Kiselman, Prolongement des solutions d'une équation aux dérivées partielles à coefficients constants, Bull. Soc. Math. France 97 (1969), 329-356.

[20] H. Komatsu, Resolutions by hyperfunctions of sheaves of solutions of differential equations with constant coefficients, Math. Ann. 176 (1968), 77-86.

[21] - Boundary values for solutions of elliptic equations, in: Proc. Internat. Conf. on Functional Analysis and Related Topics, 1969, Univ. of Tokyo Press, 1970, 107-121.

[22] —, Relative cohomology of sheaves of solutions of differential equations, in: Lecture Notes in Math. 287, Springer, 1972, 192-259.

[23] - Irregularity of characteristic elements and construction of null-solutions, J. Fac. Sci. Univ. Tokyo Sect. IA Math. 23 (1976), 297-342.

[24] - Microlocal analysis in Gevrey classes and in complex domains, in: Microlocal Analysis and Applications, Lecture Notes in Math., 1495, Springer, 1991, 161-236.

[25] H. Komatsu and T. Kawai, Boundary values of hyperfunction solutions of linear partial differential equations, Publ. Res. Inst. Math. Sci. 7 (1971/72), 95-104.

[26] G. Köthe, Dualität in der Funktionentheorie, J. Reine Angew. Math. 191 (1953), 30-49.

[27] B. Malgrange, Existence et approximation des solutions des équations aux dérivées partielles et des équations de convolution, Ann. Inst. Fourier (Grenoble) 6 (1955-56), 271-355.

[28] —, Faisceaux sur des variétés analytiques réelles, Bull. Soc. Math. France 85 (1957), 231237.

[29] F. Mantovani e S. Spagnolo, Functionali analitici reali e funzioni armoniche, Ann. Scuola Norm. Sup. Pisa (6) 18 (1964), 475-513.

[30] A. Martineau, Les hyperfonctions de M. Sato, Séminaire Bourbaki 13, 1960-61, No. 214.

[31] - Le "edge of the wedge theorem" en théorie des hyperfonctions de Sato, in: Proc. Internat. Conf. on Functional Analysis and Related Topics, 1969, Univ. of Tokyo Press, 1970, 95-106.

[32] M. Morimoto, Sur la décomposition du faisceau des germes de singularités d'hyperfonctions, J. Fac. Sci. Univ. Tokyo Sect. IA Math. 17 (1970), 215-239.

[33] C. Roumieu, Sur quelques extensions de la notion de distribution, Ann. École Norm. Sup. 77 (1960), 41-121.

[34] M. Sato, On a generalization of the concept of functions, Proc. Japan Acad. 34 (1958), 126-130 \& 604-608.

[35] -, Theory of hyperfunctions, I, J. Fac. Sci. Univ. Tokyo Sect. I 8 (1959), 139-193.

[36] - Theory of hyperfunctions, II, ibid. 8 (1960), 387-437.

[37] - Hyperfunctions and partial differential equations, in: Proc. Internat. Conf. on Functional Analysis and Related Topics, 1969, Univ. of Tokyo Press, 1970, 91-94.

[38] M. Sato, T. Kawai and M. Kashiwara, Microfunctions and pseudo-differential equations, in: Lecture Notes in Math. 287, Springer, 1973, 265-529.

[39] P. Schapira, Problème de Dirichlet et solutions hyperfonctions des équations elliptiques, Boll. Un. Mat. Ital. (4) 2 (1969), 367-372.

[40] L. Schwartz, Théorie des Distributions, Hermann, Paris 1950-51.

[41] J. Siciak, Holomorphic continuation of harmonic functions, Ann. Polon. Math. 29 (1974), 67-73. 
[42] J. Sjöstrand, Singularités analytiques microlocales, Astérisque 95 (1982).

[43] E. M. Stein, Singular Integrals and Differentiability Properties of Functions, Princeton Univ. Press, 1970. 